\section{Early cosmic background}

SIR - The existence of a black-body radiation at $3 \mathrm{~K}$ in the cosmological background is one of the essential supports of the Big Bang model of the Universe. The first measurement of the 'noise' temperature associated with this radiation is attributed to Penzias and Wilson in their famous paper published in 1965 (ref. 1), in which they announced a $3.5 \pm 1 \mathrm{~K}$ measurement at a wavelength of 7 $\mathrm{cm}$ using a detector cooled by liquid helium. In a companion paper, Dicke, Peebles, Roll and Wilkinson interpreted these results as a fossil Universe black-body radiation ${ }^{2}$. Weinberg has wondered why this cosmological background was not discovered earlier ${ }^{3}$. We can give a partial answer to this question by calling attention to a $\mathrm{PhD}$ dissertation that has until now been overlooked.

As early as 1955, E. Le Roux measured the absolute temperature of the sky as $3 \pm 2$ $\mathrm{K}$ while working at the Laboratoire de Radioastronomie in the Ecole Normale Supérieure in Paris ${ }^{4}$, in agreement with the most recent measurements from satellites and rockets ${ }^{5}$, which give a value of $2.736 \pm$ $0.017 \mathrm{~K}$. The three measurements performed by Le Roux for three different elevation angles of the antenna in 1955 led to an arithmetic mean value equal to $2.8 \mathrm{~K}$ and allowed him to show that $3 \pm 2 \mathrm{~K}$ is the only possible value to explain the consistency between the three measured values and the three corresponding equations. Moreover, the isotropy of this radiation is better than $0.5 \mathrm{~K}$, as shown by the isophotes in ref. 6 .

Le Roux attempted to compare the experimental spectrum obtained from some other available measurements with the black-body spectrum in the Rayleigh-Jeans approximation. Unfortunately, this comparison failed because of the erroneous values obtained in other papers. Nevertheless, he suggested the extragalactic origin of this radiation, although he did not compare it to early predictions of the cosmological background ${ }^{7}$.

Le Roux used an antenna which was part of a German radar system given to Professor Rocard by the British army after the Second World War. This antenna worked at $33 \mathrm{~cm}$ and its spatial detectivity was known with great precision. Hence, Le Roux knew quantitatively the contribution of every solid angle element to the antenna temperature. The detector carefully matched to the antenna was not cooled in this case and considerable noise, equivalent to a temperature of $1,450 \mathrm{~K}$, is superimposed on the useful signal. To avoid too much uncertainty in the value of the 'sky temperature', Le Roux used a clever experimental method which we have described elsewhere ${ }^{8}$. He recorded the signals obtained when the axis of the antenna was swept from the zenith to an elevation angle of $-3^{\circ}$ below the horizon. At every moment, the antenna temperature is the result of the temperatures of both the sky and the ground, with exactly known coefficients depending on the elevation angle of the antenna axis. Consequently, the antenna temperature variations from the zenith to the ground allowed Le Roux to derive the slope of the characteristics of the apparatus and to deduce several consistent measurements of the 'sky temperature'. This differential method allowed him to reach an accuracy comparable to that of Penzias and Wilson without having to cool the apparatus.

Albert Le Floch FABIEN BRETENAKER* Laboratorie d'Electronique Quantique-

Physique des Lasers,

Université de Rennes I,

Campus de Beaulieu,

35042 Rennes, Cedex, France

1. Penzias, A. A.. \& Wilson, R. W. Astrophys. J. 142, 419 (1965). 2. Dicke, R. H., Peebles, P. J. E., Roll, P. G. \& Wilkinson, D. T. Astrophys. J. 142, 414-419 (1965).

3. Weinberg, S. The First Three Minutes: A Modern View of the Origin of the Universe (Basic, New York, 1977).

Le Roux, E. thesis (Univ. Paris, 1956)

4. Le Roux, E. thesis (Univ. Paris, 1956).
5. Mather, J. C. et al. Astrophys. J. Lett. 354, L37-41 (1990). 6. Denisse, J. F., Lequeux, J. \& Le Roux. E. C. R. Acad. Sci., Paris 244B, 3030-3033 (1957).

7. Gamow, G. Nature 162, 680-682 (1948).

8. Le Floch, A. \& Bretenaker, F. C. R. Acad. Sci, Paris (in the press).

*Also at Sociêté d'Applications Générales d'Electricité et de Mécanique, 70-74 Rue de le Tour Billy, BP72, 95101 Argenteuil Cedex, France.

\section{Anti-HIV drug mechanism}

SIR - Amino acid derivatives such as $\mathrm{N}-(n-$ butyl)-deoxynojirimycin (BuDNJ), have shown exceptional promise in vitro as potential anti-HIV compounds ${ }^{1-4}$. The primary mode of action of these compounds is the inhibition of $\alpha$-glucosidases in the infected cell, leading to hyperglycosylation of the virion surface glycoprotein gp160 (refs $1,5,6)$. The exact biological consequences of hyperglycosylation and the link with the observed anti-viral activity remain uncertain. BuDNJ-treated HIV glycoproteins continue to bind to CD4 and, although the processing of gp160 to gp120 and gp41 required for viral infectivity is retarded, it is neither abolished nor reduced sufficiently wholly to explain the anti-viral effect of $\mathrm{DNJ}^{6}$. We have observed a conformational change in gp 120 following its production in the presence of BuDNJ that could help to explain the drug's action.

We expressed gp120 using recombinant baculoviruses ${ }^{7}$ in the presence or absence of BuDNJ $\left(0.5 \mathrm{mg} \mathrm{ml}^{-1}\right)$ and normalized for the amount of antigen present by quantitative western blot. The relative molecular mass of BuDNJ-treated gp120 was approximately 10,000 greater than the untreated fraction, in keeping with hyperglycosylation of the glycoprotein, but both fractions bound equally well to sCD4 and to a polyvalent antiserum against gp120 (S216), indicating no gross hyperglycosylation-induced confor-

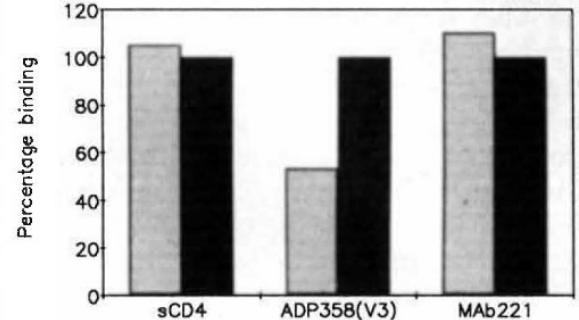

Gp120 was expressed using recombinant baculoviruses as described ${ }^{7}$ with or without the addition of $0.5 \mathrm{mg} \mathrm{ml}^{-1}$ BuDNJ. Antigen present in the supernatant of infected cells was tethered to a solid phase using the antigp120 serum D7324 (Aalto BioReagents, Dublin) and incubated with SCD4, ADP358 (an anti-V3 monoclonal antibody) or MAb 221 (a monoclonal antibody directed to the 44 amino acids at the carboxy terminus of gp120; ref. 10). Binding to gp120 was detected by incubation with the relevant conjugates (anti-CD4 or anti-mouse) and results are expressed as percentage of the binding observed with the BuDNJ-treated sample (light bars) compared to the non-BuDNJ control (heavy bars). A range of dilutions was done for each experiment but only one data point is shown here. Binding of ADP358 was similarly depressed at all dilutions tested.

mational changes in the molecule. We found the immunoreactivity of the fractions to a V3-loop-specific monoclonal antibody to be considerably different, but reactivities to a carboxy-terminal peptide-specific monoclonal antibody were unaltered (see figure).

These results suggest an alteration of the local conformation of gp 120 in the region of the hypervariable V3 loop as a consequence of BuDNJ-induced hyperglycosylation. On the basis of homology with protease inhibitors $^{8}$, it has been suggested that the V 3 loop is cleaved during viral entry and that this could be a necessary part of the infection process ${ }^{9}$. It is tempting to speculate that the altered V3 loop conformation induced by treatment with BuDNJ cannot be cleaved by the normal cellular tryptase-like enzyme, and that fusion of the viral and cellular membranes is thus prevented. Lack of syncytium formation is the observed phenotype of BuDNJtreated HIV cultures. Understanding the subtleties of the HIV infection process may yet contribute to the design of other clinically useful anti-viral agents.

IAN M. JONES

NERC Institute of Virology,

Mansfield Road,

Oxford OX1 3SR, UK

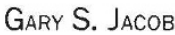

Monsanto Company,

800 North Lindbergh Blvd,

St Louis, USA

1. Gruters, R. A. et al. Nature 330, 74-77 (1987)

2. Walker, B. D. et al. Proc natn Acad SciU.S.A. 84,8120 8124 (1987)

3. Karpas, A. et al. Proc. natn. Acad. Sci. U.S.A. 85, 9229 $9233(1988)$

4. Fleet, G. W. J. et al. FEBS Lett. 237, 128-132 (1988).

5. Pal, R. et al. Intervirology 30, 27-35 (1989)

6. Ratner, L. et ai. Virology 181, 180-192 (1991).

7. Morikawa, Y etal. AIDS Res. Hum Retrovir. 6.765(1990)

8. Hattori, T. et al. FEBS Lett. 248, 48-52 (1989).

8. Hattori, T. et al. FEBS Lett. 248, 48-52 (1989).
9. Clements, G. J. et al. AlDS Res. Hum. Retrovir. 7. 3(1991) 10. Morikawa, Y. et al. J. virol. Meth. 29, 105-114 (1990). 